\title{
Antibodies to peptidoglycan in patients with spondylarthritis: a clue to disease aetiology?
}

\author{
HYDOW PARK, ${ }^{1}$ H. RALPH SCHUMACHER, 'ALLEN R. ZEIGER. ${ }^{2}$ AND \\ JAMES T. ROSENBAUM ${ }^{3}$
}

From the ${ }^{1}$ Rheumatology-Immunology Center. Veterans Administration Medical Center, Philadelphia, PA, ${ }^{2}$ Department of Biochemistry, Thomas Jefferson University, Philadelphia, PA, and ${ }^{3}$ Kuzell Institute for Arthritis Research, Medical Research Institute, San Francisco, CA, USA

SUMmARY Although the aetiology of the spondylarthritic diseases, ankylosing spondylitis and Reiter's syndrome, is obscure, a clue to the pathogenesis might be an animal model, adjuvant arthritis. Rats with this disease develop a spectrum of pathology with marked similarity to the spondylarthritides. Since peptidoglycan, a major cell wall component of most bacteria is causally implicated in adjuvant arthritis, we sought evidence that peptidoglycan exposure accompanies both Reiter's syndrome and ankylosing spondylitis. Antibodies to the D-Ala-D-Ala moiety of peptidoglycan were measured by a sensitive and specific ELISA. Antibodies were elevated significantly in patients with ankylosing spondylitis or Reiter's syndrome, but not in patients with rheumatoid arthritis or degenerative joint disease in comparison with normal controls. The findings should be considered preliminary, since only a minority of patients had increased antibody titres. However, the findings are compatible with the hypothesis that peptidoglycan is causally related to spondylarthritis. Antibodies to other moieties in the peptidoglycan molecule might be a more sensitive test for significant exposure.

The pathogenesis of spondylarthritis, including Reiter's syndrome (RS) and ankylosing spondylitis (AS), remains incompletely understood. Both diseases are strongly associated with the major histocompatibility complex antigen, HLA-B27, and RS can be provoked by a variety of infectious prodromes. $^{1}$

A possible clue to the pathogenesis of spondylarthritis is an animal model known as adjuvant arthritis. ${ }^{23}$ After an injection of killed mycobacteria into the footpad, tail, or lymph node susceptible strains of rats develop an arthropathy reminiscent of spondylarthritis in man. Similarities include sacroiliitis, syndesmophyte formation in the axial skeleton, and prominent periosteal new bone. ${ }^{23}$ In addition to the joint disease animals may develop anterior uveitis, conjunctivitis, mild bowel disease, urethritis, balanitis, and skin lesions resembling the keratodermia blennorrhagica of RS. ${ }^{2.3}$

Peptidoglycan (PG), a major cell wall component

Accepted for publication 15 March 1984.

Correspondence to Hydow Park. MD, Veterans Administration Medical Center. University and Woodland Avenues, Philadelphia. PA 19104. USA. of most bacteria, is believed to be the arthritogenic factor responsible for adjuvant arthritis in rats. ${ }^{+}$It seems plausible that PG might play a causal role in the development of spondylarthritis in man. Using a novel enzyme-linked immunosorbent assay (ELISA) specific for the D-Ala-D-Ala sequences, we report a significant elevation in antibodies to PG in patients with spondylarthritis.

\section{Materials and methods}

The study included 22 patients with AS ( 21 males, 1 female), 34 patients with RS ( 31 male, 3 female), 13 patients with degenerative joint disease (all male). 30 patients with rheumatoid arthritis $(29$ males, 1 female), and 34 normal controls (22 males, 12 females). Patients were from the clinics at the Palo Alto Veterans Administration Medical Center (VAMC), the Stanford University Medical Center, or Philadelphia VAMC. Patients with AS all had radiographic evidence of bilateral sacroiliitis and reduced motion in the axial skeleton. The diagnosis of RS was based on the clinical tetrad of arthritis. urethritis, and eye and skin diseases. All but two of 
the RS patients had at least three of these types of involvement. All patients with rheumatoid arthritis (RA) met the standard criteria of the American Rheumatism Association for classical or definite RA. Patients with degenerative joint disease (DJD) had typical radiographic and clinical presentations for that disease. Normal controls were healthy hospital personnel of the Stanford University Medical Center or the Philadelphia VAMC.

To measure antibodies to PG, synthetic PGprecursor pentapeptides (Ala- $\gamma$-D-Glu-Lys-D-AlaD-Ala) covalently linked to a random polypeptide (5) were employed as the antigen in an ELISA. Polystyrene test cups (SPIA, R \& D Lab Products, Newton Square, PA) were used as the solid phase. The test cups received $100 \mu$ l of the antigen solution (3 $\mu \mathrm{g} / \mathrm{ml}$ in carbonate buffer, $\mathrm{pH} 9 \cdot 6$ ). After an overnight incubation at $4^{\circ} \mathrm{C}$ the test cups were washed with phosphate buffered saline containing $0.05 \%$ Tween 20 (PBS/T). The subsequent incubations were all at room temperature for 45 minutes. Unknown serum samples were diluted 1:400 in $\mathrm{PBS} / \mathrm{T}$. A serum known to have a high level of anti-PG activity, stored at $-70^{\circ} \mathrm{C}$ in small aliquots, was used as a reference and tested at 1:200, 1:400, $1: 800,1: 1600$ and 1:3200 dilutions. For each sample four test cups were used, two for 'test' and two for 'control'. The control was a serum dilution containing a tripeptide, $\alpha$-Boc-Lys-D-Ala-D-Ala, as a specific antibody inhibitor, whereas the test was a serum dilution containing a tripeptide without $D$ Ala-D-Ala sequences.

These tripeptides were synthesised stepwise by classical chemical coupling procedures. ${ }^{5}$ The antigen coated test cups were first incubated with the test and control specimens. The test cups were then washed and incubated with alkaline phosphatase conjugated goat anti-human IgG (Miles Laboratories, Inc., Elkhart, IN) diluted 1:500 in PBS/T. The test cups were washed again and incubated with a substrate solution $(1 \mathrm{mg} / \mathrm{ml} p$-nitrophenyl phosphate) in a dark moist chamber. The contents of each test cup were then mixed with $1.5 \mathrm{ml} 1 \mathrm{~N}$ $\mathrm{NaOH}$ solution and transferred to test tubes. The absorbances were measured at $405 \mathrm{~nm}$ in a spectrophotometer. The mean absorbance of the two controls was subtracted from that of the tests to obtain a net absorbance. The net absorbances of the serial dilutions of the reference serum were plotted arithmetically against antibody levels. The antibody levels of unknown samples were estimated from this graph. The levels were expressed as a percent of the level of the reference serum.

Statistical analyses were based on a one-tailed Student's $t$-test or a $\chi^{2}$ analysis. The difference was considered significant when $\mathrm{p}<0.05$.

\section{Results}

The antibody levels of the patients and controls are shown in Fig. 1. Thirty-four normal individuals had a mean of $14.7 \pm 2.4 \%$ (mean \pm SEM). There was no significant difference in antibody levels between males and females (Fig. 1). The 13 patients with degenerative joint disease (DJD) had a mean of 12.0 $\pm 3 \cdot 3 \%$, which was not significantly different from that of the normal group. The mean antibody level of 30 RA patients $(18 \cdot 8 \pm 4 \cdot 1 \%)$ was higher, but not significantly different from that of the normal group.

By contrast the mean antibody level of 22 AS patients $(51.6 \pm 19.0 \%)$ was significantly higher than that of the normal group $(p<0.025)$, the normal and DJD (combined) group $(\mathrm{p}<0.005)$, the RA group $(\mathrm{p}<0.025)$, and the RS group $(\mathrm{p}<0.05)$. The mean antibody level of $34 \mathrm{RS}$ patients $(22.4 \pm$ $4.5 \%$ ) was not significantly higher than that of the normal group or the RA group, but was significantly higher than that of the normal and DJD (combined) group $(\mathrm{p}<0.05)$. If patients with spondylarthritis are considered together as a single disease entity, the mean antibody level $(33.9 \pm 8 \cdot 1 \%)$ was significantly higher than that of the normal group $(\mathrm{p}<0.05)$ or the normal and DJD (combined) group $(\mathrm{p}<0.025)$.

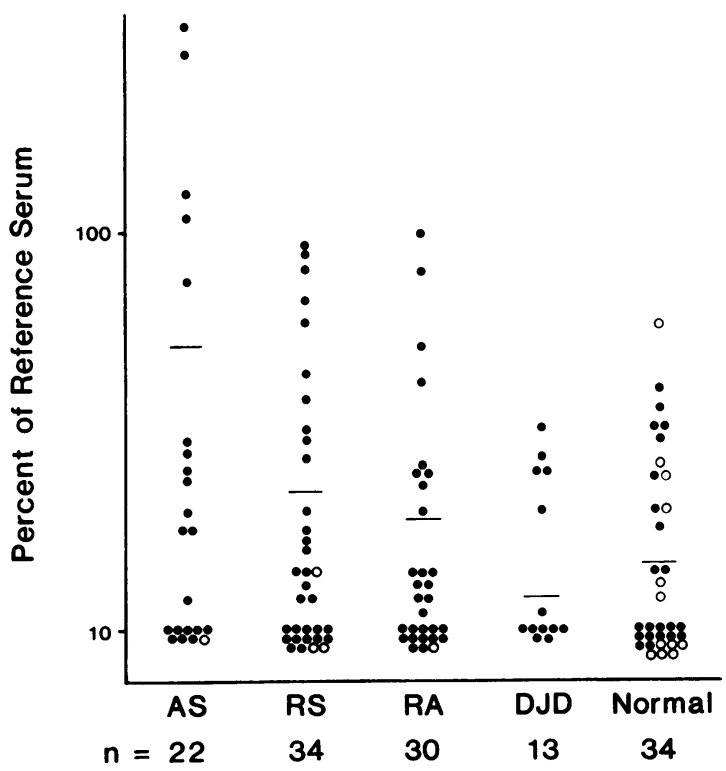

Fig. 1 Distribution of serum anti-PG antibody levels in patients with ankylosing spondylitis $(A S)$, Reiter's syndrome $(R S)$, rheumatoid arthritis $(R A)$, degenerative joint disease (DJD), and normal controls. The horizontal bars indicate the mean anti-PG antibody levels. Open and closed circles represent female and male subjects respectively. 
To control for the possibility that the two highest values in the AS group were unduly skewing the data, a $\chi^{2}$ analysis was also performed. In a comparison the AS with the normal group the finding of 5 of the 22 values more than 2 standard deviations outside the normal range was statistically significant by $\chi^{2}$ analysis $(p<0 \cdot 05)$. The RS population also differed significantly from the normals by $\chi^{2}$ analysis (6 of 34 greater than 2 standard deviations beyond the normal range, $\mathrm{p}<0 \cdot 05$ ). The RA and DJD groups did not differ significantly from the normals by $\chi^{2}$ analysis.

HLA typing had been performed in 10 AS patients (all B27 positive) and 18 RS patients, 12 of whom were B27 positive. The mean antibody level of the six B27 negative patients $(34 \cdot 6 \pm 2 \cdot 4 \%)$ did not differ significantly from that of the B27 positive groups $(27.6 \pm 8 \cdot 2 \%)$.

The AS patients with high antibody levels were carefully checked and showed no evidence of active bacterial infection and no correlation between antibody level and disease activity. Three RS patients who developed disease after Gram-negative bacterial dysentery and one AS patient who had associated inflammatory bowel disease did not have raised antibody levels.

\section{Discussion}

This study demonstrates a statistically significant increase in antibody titre to peptidoglycan in patients with spondylarthritis. Low titres of these antibodies are found in normal sera, while elevated titres are induced by active bacterial infections. ${ }^{6}$ Elevated antibody titres to $P G$ have been reported in RA, but this finding has been questioned. Elevated titres have also been reported in rheumatic fever and juvenile rheumatoid arthritis. ${ }^{78}$ Elevated anti-PG antibody levels in spondylarthritis are not the likely result of an age or sex bias in the population, elevated total IgG levels, or circulating immune complexes. No significant correlation was noted between age and antibody level in these patients $(r=0 \cdot 09, p<0 \cdot 2)$. In addition the AS group differed from other groups representing either younger or older people. The mean ages of the AS, RS, RA, DJD, and normal groups were $42,35,56$, 64,40 , respectively. The ELISA method was designed to eliminate the problem of non-specific binding of IgG molecules by using the 'control'. The RA group provided an additional control for bias from elevated IgGs or immune complexes. It also seems unlikely that medication accounts for the elevation in antibody titres, since the patients in both the RA and DJD groups received similar anti-inflammatory drugs relative to the AS and RS patients. Oral penicillin results in soluble PG appearing in the urine. ${ }^{9}$ Conceivably, antibiotic therapy for urethritis results in PG exposure, but this explanation cannot account for the findings in AS. We cannot readily explain the apparent difference between AS and RS patients, but both groups differ significantly from normals.

If an abnormal exposure to PG is causally related to spondylarthritis, how does this occur? Gramnegative bacterial dysentery might cause an abnormal exposure to PG released either from pathogenic bacteria or indigenous bacteria of the intestinal tract. Systemic absorption of Gram-negative bacterial cell wall has been reported during shigellosis. ${ }^{10}$ Exposure to PG might similarly occur during urethritis, since the urethra is colonised with a normal flora, and inflammation could promote absorption of bacterial cell wall products. Mycoplasma and chlamydia, two types of organisms associated with the urethritis prodrome of RS, are not known to contain PG. Occult prostatitis, reported to be a frequent concomitant of AS, ${ }^{11}$ might cause an abnormal exposure to PG. Some studies have suggested a relationship between intestinal klebsiella and AS. ${ }^{12}{ }^{13}$ In one report a positive stool culture for klebsiella in AS patients was strongly associated with the activity of the disease. ${ }^{13}$

If exposure to PG is sausally related to spondylarthritis, why are antibody levels elevated in only a minority of affected patients? There are several possible explanations for this. First, although the D-Ala-D-Ala moiety of the PG molecule is immunodominant among Gram-positive bacteria, it is not a prominent antigen in the Gram-negative bacterial cell wall. ${ }^{14}$ Epidemiologically, Gramnegative bacteria provoke RS, and our study might easily have missed serological evidence of exposure to these organisms. This is certainly suggested by the data on three individuals with postdysenteric RS. Secondly, again by analogy with the animal model, an immune response to $P G$ itself is not essential for adjuvant disease to develop. ${ }^{15}$ Thirdly, PG might be only one of several possible adjuvants that might trigger spondylarthritis.

These data should be seen as a preliminary attempt to link exposure to PG with spondylarthritis. The statistical association is significant but weak and not proof of causality. Obviously not all patients with elevated anti-PG antibodies develop spondylarthritis. Multiple factors could determine the arthritogenic potential of $\mathrm{PG}$, including the source of PG, the route of exposure, the dose, the duration of exposure, and the genetic background of the responder. Nevertheless, significant elevation of anti-PG antibody levels in some patients with spondylarthritis indicates abnormal exposure or 
response to this antigen. We speculate that $P G$ is an adjuvant that may trigger spondylarthritis in man. Further studies are needed to evaluate the response in patients with spondylarthritis to PG moieties prominent among Gram-negative bacteria.

Supported in part by a grant (Dr Park) from the Arthritis Foundation Eastern Pennsylvania Chapter and a New Investigator Research Award (Dr Rosenbaum) from the National Institutes of Health, AM 31076.

\section{References}

1 Rosenbaum J T. Why HLA-B27: an analysis based on two animal models. Ann Intern Med 1981; 94: 261-3.

2 Pearson C M, Chang Y H. Adjuvant disease: pathology and immune reactivity. Ann Rheum Dis 1979; 38: (suppl): 102-9.

3 Pearson C M, Waksman B H, Sharp J T. Studies of arthritis and other lesions induced in rats by injection of mycobacterial adjuvants. V. Changes affecting the skin and mucous membranes. Comparison of the experimental process with human disease. J Exp Med 1961; 113: 485-510.

4 Kohashi O, Pearson C M, Watanabe Y, Kotani S, Koga T. Structural requirements for arthritogenicity of peptidoglycans from Staphylococcus aureus and Lactobacillus plantarum and analagous compounds. J Immunol 1976; 116: 1635-1639.

5 Zeiger A R, Maurer P H. Immunochemistry of a synthetic peptidoglycan-precursor pentapeptide. Biochemistry 1973; 12: 3387-94.
6 Zeiger A R, Tuazon C U, Sheagren J N. Antibody levels to bacterial peptidoglycan in human sera during the time course of endocarditis and bacteremic infections caused by Staphylococcus aureus. Infect Immun 1981; 33: 795-800.

7 Pope R M, Rutstein J E, Straus D C. Detection of antibodies to streptococcal mucopeptide in patients with rheumatic disorders and normal controls. Int Arch Allergy Appl Immunol 1982; 67: 267-74.

8 Heymer B, Schleifer K-H, Read S, Zabriskie J B, Krause R M. Detection of antibodies to bacterial cell wall peptidoglycan in human sera. J Immunol 1976; 117: 23-6.

9 Park H, Schumacher H R, Zeiger A R. Detection of soluble peptidoglycan in urine following penicillin administration. Infect Immun 1984; 43: 171-5.

10 Koster S, Levin J, Walker L, et al. Hemolytic-uremic syndrome after shigellosis. Relation to endotoxemia and circulating immune complexes. N Engl J Med 1978; 298: 927-33.

11 Mason R M, Murray R S, Oates J K, Young A C. Prostatitis and ankylosing spondylitis. $\mathrm{Br}$ Med $J$ 1958; i: 748-51.

12 Seager K, Bashir H V, Geczy A, Edmonds J, DeVere T. Evidence for a specific B27-associated cell surface marker on lymphocytes of patients with ankylosing spondylitis. Nature 1979; 277: 68-70.

13 Ebringer R W, Cawdell D R, Cowling P, Ebringer A. Sequential studies in ankylosing spondylitis. Association of Klebsiella pneumoniae with active disease. Ann Rheum Dis 1978; 37: 146-51.

14 Nguyen-Huy H, Nauciel C, Wermuth C G. Immunochemical study of the peptidoglycan of Gram-negative bacteria. Eur $J$ Biochem 1976; 66: 79-84.

15 Nagao S, Tanaka A. Muramyl dipeptide-induced adjuvant arthritis. Infect Immun 1980; 28: 624-5. 